\title{
Bio-inspired Networking: From Theory to Practice
}

\author{
Falko Dressler* and Ozgur B. Akan ${ }^{\dagger}$ \\ ${ }^{*}$ Computer Networks and Communication Systems, Dept. of Computer Sciences, University of Erlangen, Germany \\ Email: dressler@informatik.uni-erlangen.de \\ ${ }^{\dagger}$ Dept. of Electrical and Electronics Engineering, Koc University, Istanbul, Turkey \\ Email: akan@ku.edu.tr
}

\begin{abstract}
Bio-inspired networking techniques have been investigated since more than a decade. Findings in this field have fostered new developments in networking, especially in the most challenging domains such as handling large scale networks, their dynamic nature, resource constraints, heterogeneity, unattended operation, and robustness. Even though this new research area started with highly theoretical concepts, it can be seen that there is also practical impact. This article aims to give an overview to the general field of bio-inspired networking, introducing the key concepts and methodologies. Selected examples that outline the capabilities and the practical relevance are discussed in more detail. The presented examples outline the activities of a new community working on bio-inspired networking solutions, which is converging and becomes visible in term of the provided astonishingly efficient solutions.
\end{abstract}

\section{INTRODUCTION}

After a decade of bio-inspired networking research, we see many very successful application examples that either directly improve specific networking-related operations or simply foster novel research projects to reinvestigate what we thought of being common knowledge in engineering. In fact, when we look carefully into nature, it is clearly observed that the dynamics of many biological systems and laws governing them are based on a surprisingly small number of simple generic rules which yield collaborative yet effective patterns for resource management and task allocation, social differentiation, synchronization (or de-synchronization) without the need for any externally controlling entity. For example, by means of these capabilities, billions of blood cells which constitute the immune system can protect an organism from pathogens without any central control of the brain [1]. Similarly, an entire organism is autonomously maintained in a relatively stable equilibrium state via a major functionality, i.e., homeostasis, for the operation of vital functions without any need for a central biological controller [2]. The task allocation process in the insect colonies is collaboratively decided and performed according to the willingness of an individual such that the overall task is optimized with a global intelligence comprised of simple individual responses [3].

At the same time, communication and management aspects in networking are becoming even more challenging in future networking domains ranging from nanoscale communication networks [4] to InterPlaNetary Internet [5]. Technical challenges include the management of thousands and millions of inter-networking devices that have to be organized using scare resources and disruptive communication channels. In spite of these limitations, the networking community is developing

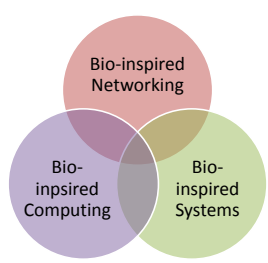

Fig. 1. Bio-inspired networking in relation to the entire field of bio-inspired approaches

astonishing technical solutions, in many cases inspired by selforganization mechanisms inherently existing in Nature.

This paper is intended to review the state-of-the-art of the emerging domain of bio-inspired networking and its more practical relevance that has been established in the last decade. Using selected application examples, we highlight both the fascinating simplicity of the underlying rules and mechanisms as well as the sophisticated overall behavior of such bioinspired solutions to networking problems.

The paper is organized into four sections. We first outline the main concepts and ideas of bio-inspired networking solutions including a list of the most prominent challenges in networking in Section II. Selected examples are discussed in more detail in Section III. Future research challenges and directions are outlined in Section IV. We finally draw some conclusions in Section V.

\section{Bio-inspired Networking: Overview of Challenges and Fundamental ANALOgies}

We concentrate on the application domains of bio-inspired solutions to problems related to communications and networking. As shown in Figure 1, three main areas of bio-inspired research can be distinguished:

- Bio-inspired computing represents a class of algorithms focusing on efficient computing, e.g., for optimization processes and pattern recognition.

- Bio-inspired systems constitute a class of system architectures for massively distributed and collaborative systems, e.g., for distributed sensing and exploration.

- Bio-inspired networking is a class of strategies for efficient and scalable networking under uncertain conditions, e.g., for autonomic organization in largely distributed systems.

Even though bio-inspired computing and system design have already become widely visible, for example, neuronal networks have been thoroughly studied and successfully applied in many places, the application domain of bio-inspired 
TABLE I

CATEGORIZATION OF BIOLOGICAL PHENOMENA AND NETWORKING ALGORITHMS MIMICKING THESE CONCEPTS

\begin{tabular}{ll}
\hline Biological principle & Application fields in networking \\
\hline $\begin{array}{l}\text { Swarm Intelligence and } \\
\text { Social Insects }\end{array}$ & $\begin{array}{l}\text { distributed search and optimization; routing in computer networks, especially } \\
\text { in MANETs, WSNs, and overlay networks; task and resource allocation }\end{array}$ \\
\hline Firefly Synchronization & robust and fully distributed clock synchronization \\
\hline $\begin{array}{l}\text { Activator-Inhibitor } \\
\text { Systems }\end{array}$ & $\begin{array}{l}\text { (self-) organization of autonomous systems; distributed coordination; continuous } \\
\text { adaptation of system parameters in highly dynamic environments }\end{array}$ \\
\hline $\begin{array}{l}\text { Artificial Immune } \\
\text { System }\end{array}$ & network security; anomaly and misbehavior detection \\
\hline Epidemic Spreading & $\begin{array}{l}\text { content distribution in computer networks (e.g. in DTNs); overlay networks; } \\
\text { analysis of worm and virus spreading in the Internet }\end{array}$ \\
\hline $\begin{array}{l}\text { Cellular Signaling } \\
\text { Networks }\end{array}$ & $\begin{array}{l}\text { coordination and control in massively distributed systems; programming of } \\
\text { network-centric operating sensor and actor networks }\end{array}$ \\
\hline
\end{tabular}

networking is a rather new one that is emerging from early studies into well-understood and carefully investigated solutions.

There exist many challenges for the realization of the existing and the envisioned next generation network architectures. Among others, three examples should be named: The dynamic nature of mobile ad hoc networks and cognitive radio networks in terms of node behaviors, traffic and bandwidth demand patterns, channel and network conditions need to be handled using completely new approaches. Thus, communication techniques that are inherently adaptive to the highly dynamic network conditions must be developed for the next-generation network architectures.

A second major challenge is the demand for infrastructureless and autonomous operation. As network dimensions are amplified both spatially and in terms of the number of nodes, centralized control of communication becomes impractical. On the other hand, some networks are by definition free from infrastructure such as wireless ad hoc networks, Delay Tolerant Networks (DTNs), Wireless Sensor Networks (WSNs). At the same time, communication networks are subject to failure either by device and link malfunction or misuse of their capacity. Considering the dynamic nature and lack of infrastructure, networks must have capabilities of self-organization [6], selfevolution, and survivability to be able to continuously provide their services.

Furthermore, communication and networking in micro and nano scales are imperative to enable micro and nano devices to cooperate, and hence, collaboratively realize certain common complex tasks which cannot individually be handled. On the other hand, conventional communication technologies such as electromagnetic wave, acoustic, are inapplicable at these scales due to antenna size and channel limitations. Furthermore, the communication medium and channel characteristics also show important deviations from the traditional cases due to the rules of physics governing these scales. Effective, practical, and naturally existing communication paradigms must be researched and adopted for networking at these scales.

These challenges may ultimately be addressed by bioinspired solutions since similar problems and their naturally evolved biological solution approaches also exist for these networking paradigms. In fact, as a result of millions of years of evolution, biological systems and processes have intrinsic appealing characteristics [7]. Among others [8], they are
- adaptivity to the varying environmental circumstances,

- robustness and resilient to the failures caused by internal or external factors,

- ability to achieve complex behaviors on the basis of a usually limited set of basic rules,

- ability to learn and evolve itself when new conditions are applied,

- effectiveness management of constrained resources with an apparently global intelligence larger than the superposition of individuals,

- ability to self-organize in a fully distributed fashion, collaboratively achieving efficient equilibrium,

- and survivability despite harsh environmental conditions due to its inherent and sufficient redundancy.

These characteristics constitute the basis for different levels of inspiration by biological systems towards the development of different approaches and algorithms at each of the networking layers for efficient, robust and resilient communication and information networks [8]. Therefore, in order to keep pace with the evolution in networking technologies, many researchers, members of this very young research community, are currently engaged in developing innovative design paradigms inspired by biology in order to address the networking challenges of the existing and envisioned next-generation information systems as outlined above.

The common rationale behind this effort is to capture the governing dynamics and understand the fundamentals of biological systems in order to devise new methodologies and tools for designing and managing communication systems and information networks that are inherently adaptive to dynamic environments, heterogeneous, scalable, self-organizing, and evolvable. Looking from biological principles, several application domains in networking can be distinguished. Table I summarizes the biological domains that are, together with specific examples of successful application to networking, detailed in Section III.

\section{Selected Examples: Simple Concepts, Complex SOLUTIONS}

The objective of this section is to discuss the concepts and application domains of selected examples of bio-inspired networking approaches. As can be seen from these examples, most of the solutions - if appropriately modeled - are built on simple concepts and rules. The underlying mathematical 
models or architectural concepts can easily be understood and implemented. However, the resulting behavior of the overall system composed of many of such simple components, due to its nonlinear coupling, quickly becomes much too complex for analytical evaluation.

In the following, selected examples are discussed that represent well understood biological mechanisms, even though some aspects might need to be further elaborated in detail. This list is not meant to be comprehensive and to completely represent all approaches in the domain of bio-inspired networking. However, we selected a number of techniques and methods for more detailed presentation that clearly show advantages in fields of communication networks. Further examples and references can be found in [8].

\section{A. Modeling and Engineering}

The modeling and systematic development of new approaches slightly deviate from classical engineering processes. In early papers on bio-inspired solutions (unfortunately, even in some recent publications), bio-inspired networking has been considered as attempts to present (engineering) technical solutions solely based on some similarities to biological counterparts without actually investigating the key advantages or objectives of the biological systems.

In fact, a careful look at the more mature studies in the field reveals that three common steps are always necessary for developing bio-inspired methods that have a remarkable impact in the domain under investigation, i.e.,

1) Identification of analogies - which structures and methods seem to be similar,

2) Understanding - detailed modeling of realistic biological behavior,

3) Engineering - model simplification and tuning for technical applications.

These primary principles of investigating and exploiting biological inspirations are depicted in Figure 2. First, analogies between biological and technical systems such as computing and networking systems must be identified. It is especially necessary that all the biological principles and their governing dynamics are understood properly, which is often not yet the case in biology. Secondly, models that capture the biological behavior must be created to be later used to develop the technical solution. The translation from biological models to the model describing bio-inspired technical systems is a pure engineering step. Finally, the model must be simplified and tuned for the technical application.

\section{B. Ant Colony Optimization}

Ant Colony Optimization (ACO) is one of the best analyzed and most frequently applied branches in the field of swarm intelligence. ACO is based on the observation of the collective foraging behavior of ants [3]. This can be modeled as a massively distributed self-organized system, which is typically made up of a population of simple agents interacting locally with one another and with their environment [6]. It is well known that ants are able to solve quite complex tasks

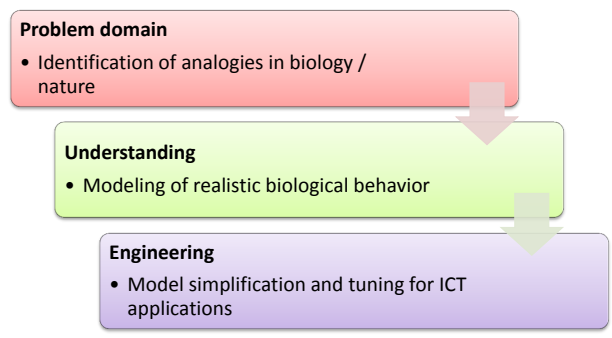

Fig. 2. Modeling and engineering process

by simple local means, relying only on indirect interaction between individuals. For example, pheromone trails are used for efficient foraging. Ants are "grand masters" in search and exploration.

Conceptually, ants perform a random search (random walk) for food. The way back to the nest is marked with a pheromone trail. If successful, the ants return to the nest (following their own trail). While returning, an extensive pheromone trail is produced pointing towards the food source. Further ants are recruited that follow the trail on the shortest path towards the food. The ants, therefore, communicate based on environmental changes (pheromone trail). This communication type is also known as stigmergy.

The complete ACO algorithm is described in [9]. The most important aspect is the transition probability $p_{i j}$ for an ant $k$ to move from $i$ to $j$. This probability represents the routing information for the exploring process, i.e.,

$$
p_{i j}^{k}=\left\{\begin{array}{cc}
\frac{\left[\tau_{i j}(t)\right]^{\alpha} \times\left[\eta_{i j}\right]^{\beta}}{\sum_{l \in J_{i}^{k}}\left[\tau_{i l}(t)\right]^{\alpha} \times\left[\eta_{i l}\right]^{\beta}}, & \text { if } j \in J_{i}^{k} \\
0, & \text { otherwise }
\end{array}\right.
$$

Each move depends on the following parameters:

- $J_{i}^{k}$ is the tabu list of not yet visited nodes, i.e., by exploiting $J_{i}^{k}$, an ant $k$ can avoid visiting a node $i$ more than once.

- $\eta_{i j}$ is the visibility of $j$ when standing at $i$, i.e., the inverse of the distance.

- $\tau_{i j}$ is the pheromone level of edge $(i, j)$, i.e., the learned desirability of choosing node $j$ and currently at node $i$.

- $\alpha$ and $\beta$ are adjustable parameters that control the relative weight of the trail intensity $\tau_{i j}$ and the visibility $\eta_{i j}$, respectively.

After completing a tour, each ant $k$ lays a quantity of pheromone $\Delta \tau_{i j}^{k}(t)$ on each edge $(i, j)$ depending on the length of the tour done by ant $k$ at iteration $t$.

Dynamics in the environment are explicitly considered by the ant foraging scheme. The pheromone slowly evaporates. Thus, if foraging ants are no longer successful, the pheromone trail will dissolve and the ants continue with their search process. Additionally, randomness is also a strong factor during successful foraging. A number of ants will continue the random search for food. This adaptive behavior leads to an optimal search and exploration strategy. This effect is provided by the pheromone update rule, where $\Delta \tau_{i j}(t)=\sum_{k=1}^{m} \Delta \tau_{i j}^{k}(t)$ (the decay is implemented in form of a coefficient $\rho$ with 

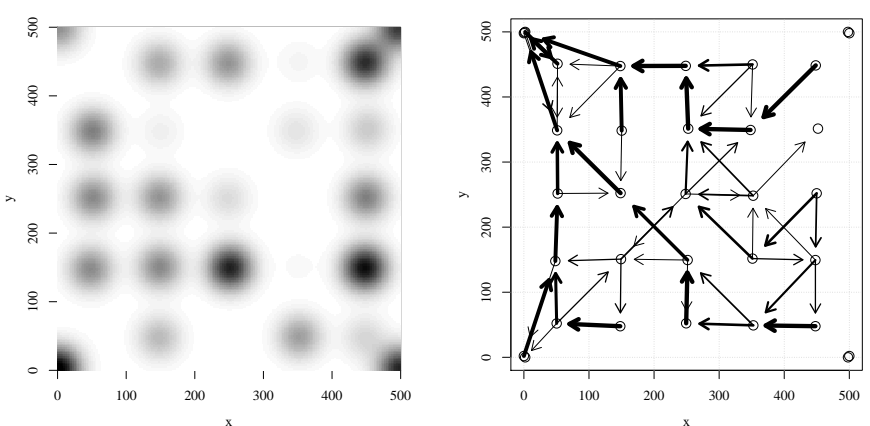

Fig. 3. ACO-based task allocation with integrated route optimization [11]

$0 \leq \rho<1)$, i.e.,

$$
\tau_{i j}(t) \leftarrow(1-\rho) \times \tau_{i j}(t)+\Delta \tau_{i j}(t)
$$

According to [9], the total number of ants $m$ is an important parameter of the algorithm. Too many ants would quickly reinforce suboptimal tracks and lead to early convergence to bad solutions, whereas too few ants would not produce enough decaying pheromone to achieve the desired cooperative behavior. Thus, the decay rate needs to be carefully controlled.

Perhaps the best known application example of ACO in networking is the AntNet [10] routing protocol. In particular, so called agents are used to concurrently explore the network and exchange collected information in the same way as ants explore the environment. The communication among the agents is indirect, following the stigmergy-based approach, and mediated by the network itself.

Based on the same concepts, integrated task allocation and routing in Sensor and Actor Networks (SANETs) has been investigated [11]. The proposed architecture is completely based on probabilistic decisions. During the lifetime of the SANET, all nodes maintain and adapt a probability $P(i)$ to execute a task $i$ out of a given set. Reinforcement strategies are exploited to optimize the overall system behavior. Heterogeneity is inherently supported. Therefore, the task lists of different agents will be different. The probability to chose a task $P(i)$ can now be calculated according to the shown ACO formulas. Furthermore, routing is performed similar to AntNet except for one major difference. In order to support the task specific communication, the routing table is extended to cover different forwarding probabilities for the defined tasks. Figure 3 shows results from selected simulations. A number of tasks were injected into a 25 nodes network. As can be seen, the nodes optimize themselves for particular tasks (left figure) and the routing layer enforces paths optimal for the current task allocation (right figure).

\section{Artificial Immune System}

The term Artificial Immune System (AIS) belongs to a terminology that refers to adaptive systems inspired by theoretical and experimental immunology with the goal of problem solving [12]. The primary goal of an AIS, which is inspired by the principles and processes of the mammalian immune system, is to efficiently detect changes in the environment or deviations (non-self) from the normal system behavior (self)

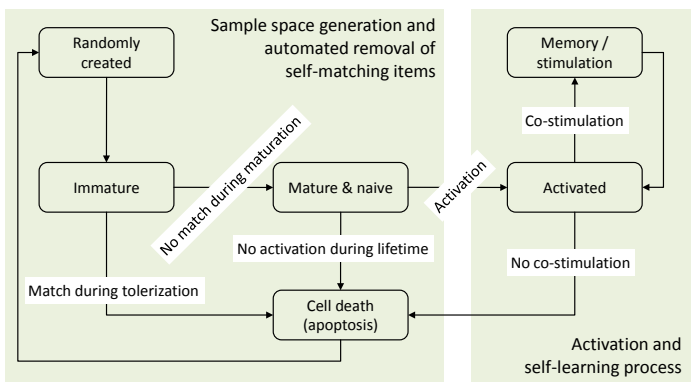

Fig. 4. Conceptual model of AIS properties

TABLE II

RELATIONSHIP BETWEEN IMMUNE SYSTEM AND WIRELESS SENSOR NETWORKS (WSN) [15].

\begin{tabular}{c|c}
\hline Immune System & Wireless Sensor Networks (WSN) \\
\hline \hline B-cells & Sensor nodes \\
\hline Antibody & Sensor data \\
\hline Antibody density & Reporting frequency rate $f$ \\
\hline T-cells & Rate control parameter \\
\hline Pathogen & Event source \\
\hline Antigen & Estimation distortion \\
\hline Natural extinction & Packet loss \\
\hline
\end{tabular}

in complex problems domains, and to automatically memorize these characteristics. An AIS basically consists of three parts, which have to be worked out in the immune engineering process [12]:

- Representations of the system components, i.e., the mapping of technical components to antigens and antibodies.

- Affinity measures, i.e., mechanisms to evaluate interactions (e.g., stimulation pattern and fitness functions) and the matching of antigens and antibodies.

- Adaptation procedures to incorporate the system's dynamics, i.e., genetic selection.

The underlying concepts are outlined in Figure 4. Detectors are randomly generated to statistically cover the entire measurement range. These new detectors can basically identify either system characteristics (self) or possible deviations (nonself). In order to eliminate the self detectors, an early tolerization process is used. All others enter the detection system until their lifespan has been reached. Successful detections are memorized allowing an accelerated response the next time.

Early approaches showing the successful application of an AIS in computer and communication systems have been presented in [13]. Meanwhile, a number of frameworks are available. Focusing on the design phase of an AIS, an immune engineering framework is proposed in [12]. A similar conceptual frameworks for Artificial Immune Systems for generic application in networking has been presented in [14]. Again, the three design steps have been emphasized: representation, selection of appropriate affinity measures, and development of immune algorithms.

An application of an immune system based distributed node and rate selection in sensor networks has been proposed in [15]. Sensor networks and their capabilities, in particular their transmission rate, are modeled as antigens and antibodies as 
outlined in Table II. The distributed node and rate selection (DNRS) algorithm for event monitoring and reporting is realized by means of B-cell stimulation, i.e., appropriate source node and rate selection.

Inspired by the B-cell stimulation mechanism, the source node selection is provided by the following influences: (1) the affinity between a sensor node (B-cell) and event source (pathogen), (2) the affinity between the sensor node and its uncorrelated neighbor nodes (stimulating B-cells), and (3) the affinity between the sensor node and its correlated neighbor nodes (suppressing B-cells). DNRS selects each sensor node for which the summation of these three influences is greater than a predefined threshold as a source node.

After the selection of source nodes considered as stimulated B-cells in immune system, DNRS also enables these source nodes to separately determine their reporting frequency rates. Similar to stimulated B-cells that secrete antibody molecules to eliminate antigen molecules in immune system, source nodes makes data transmission to minimize event signal distortion at the sink. Based on a rate control parameter, $F_{i}(t)$, derived from the principles of antibody secretion mechanism in immune system, the new reporting frequency rate of source node $i$ at time $t+1$, i.e., $f_{i}(t+1)$, is determined as

$$
f_{i}(t+1)=\frac{1}{1+e^{\left[0.5-F_{i}(t)\right]}}
$$

DNRS allows each source node $i$ to separately determine $f_{i}(t+1)$ by computing $F_{i}(t)$ based on the interaction with its environment. This makes DNRS highly adaptive and distributed algorithm that does not need any central controller or any predefined static frequency update rules.

\section{Cellular Signaling Cascades}

The term signaling describes the interactions between single signaling molecules [16]. Such communication, also known as signaling pathways [17], is an example for very efficient and specific communication. Cellular signaling occurs at multiple levels and in many shapes. Briefly, cellular interactions can be viewed as processing in two steps. Initially, an extracellular molecule binds to a specific receptor on a target cell, converting the dormant receptor to an active state. Subsequently, the receptor stimulates intracellular biochemical pathways leading to a cellular response [17].

A key challenge for biology is to understand the structure and the dynamics of the complex web of interactions that contribute to the structure and function of a living cell. In order to uncover the structural design principles of such signaling networks, network motifs have been defined as patterns of interconnections occurring in complex networks at numbers that are significantly higher than those in randomized networks [18].

A number of approaches have been discussed using artificial signaling networks for networking applications. Most of this work is targeting programming schemes for massively distributed systems such as sensor networks. An example is the Rule-based Sensor Network (RSN) concept, a light-weight programming scheme for SANETs [19]. It is based on an architecture for data-centric message forwarding, aggregation,

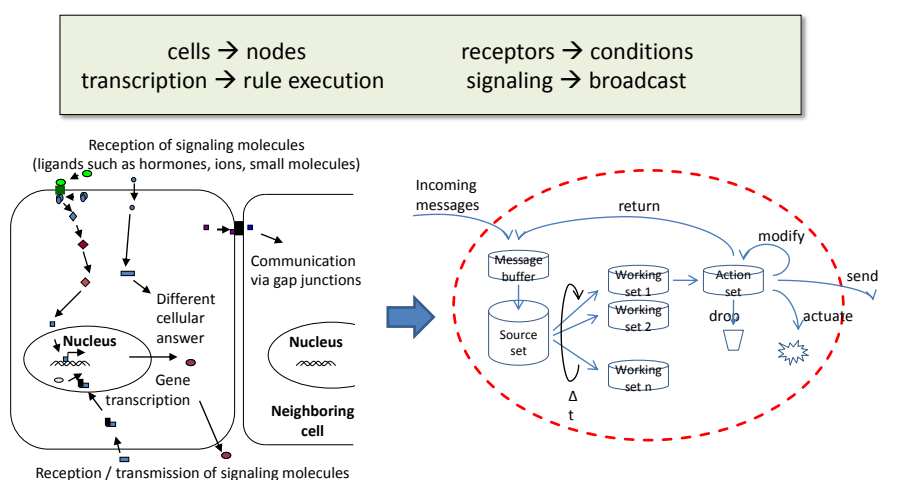

Fig. 5. Modeling of cellular signaling cascades and application to programming sensor and actor networks [19]

and processing, i.e., using self-describing messages instead of network-wide unique address identifiers. It has been shown that quite complex systems can be successfully programmed using this system supporting also concepts of light-weight reprogramming resource-restricted sensor nodes [19].

Figure 5 outlines the working behavior of a single RSN node. On the left hand side, the signaling process within a single cell is depicted. Several transformations of received information particles, e.g., proteins, finally lead to the cellular response. Similarly, RSN stores received messages in a buffer. A rule interpreter is then started periodically (after a fixed $\Delta t$ ) or after the reception of a new message. An extensible and flexible rule system is used to evaluate received messages and to provide the basis for the node programming scheme. The specific reaction on received data is achieved by means of predicate-action sequences of the form if PREDICATE then \{ ACTION \}.

First, all messages matching the predicate are stored in so called working sets. Finally, the specified action is executed on all the messages in the set. Using such rule-sets, complex and dynamic behavior can be modeled. Examples are event monitoring applications in sensor networks or target tracking under energy constraints. In biological systems such behavior can be modeled (or studied) using signaling networks and repetitive patterns, or motifs.

The period of RSN execution $\Delta t$ has been identified as a key parameter for controlling the reactivity vs. energy performance of the entire RSN-based network. Basically, the duration of messages stored in the local node introduces an artificial perhop delay. The optimal value for $\Delta t$ affects the aggregation quality vs. real-time message processing. A promoter-inhibitor system has successfully been applied to solve this issue [20].

Another approach for a metabolistic execution model for communication protocols was named Fraglets [21]. Similar to RSN, this model is also based on the concept of datacentric communication. Furthermore, the execution relies on the unification of code and data, featuring a single unit called "fraglets" that are operands as well as operators. Fraglets have surprising strong ties to formal methods as well as to molecular biology. At the theory level, fraglets belong to string rewriting systems. In particular, fraglets are symbol strings $\left[s_{1}: s_{2}: \ldots\right.$ tail $]$ that represent data and/or logic, where tail 
is a (possibly empty) sequence of symbols. Each node in the network has a fraglet store to which incoming fraglets are added. The node continuously examines the fraglet store and identifies which fraglets need to be processed. Simple actions lead to transformations of a single fraglet. More complex actions combine two fraglets. If several actions are possible at a time, the system randomly picks one action, atomically removes the involved fraglets from the store, processes them, and puts potential results back into the store [21].

Using the fraglet system, network-centric operations can be specified to be executed by participating nodes after reception of a specific fraglet. A simple example of a fraglet program is the following confirmed-delivery protocol (CDP) that transfers received $[c d p: d a t a]$ fraglets from $A$ to $B$, with per packet acknowledgments [21]:

$$
\begin{aligned}
& { }_{A}[\text { match } P: \text { cdp }: \text { send }: B: \text { deliver }] \\
& { }_{B}[\text { match } P: \text { deliver }: \text { split }: \text { send }: A: \text { ack }: *]
\end{aligned}
$$

\section{Discussion And SOME Open RESEARCH DIRECTIONS}

In this section, we summarize the presented concepts of bio-inspired networking by discussing some open research challenges.

\section{A. Investigation of Artificial Models}

It needs further to be mentioned that our bio-inspired systems are already being studied by the biologists to learn more about the behavioral patterns in nature. Thus, the loop closes from technical applications to biological systems as well. This constitutes a very promising and largely unexplored research field. Two examples are briefly outlined in the following to highlight both the relevance and the potentials of this research field:

- Learning robots are frequently programmed using some cognitive capabilities and social group behavior. On the other hand, robots can be used as models of specific animal systems to test hypotheses regarding the control of behavior. Implementing selected learning and control techniques, and testing those against real environments can reveal interesting aspects of the used control systems. This often also provides insight into the true nature of the problem and may lead to novel hypotheses for animal behavior [22].

- Investigations of Artificial Immune Systems not only allow to develop more sophisticated technical solutions but also helps to improve the underlying theoretical models of the immune system. The results can be directly applied to study yet unknown aspects of auto-immune reactions and other dysfunctions. Also, the influence of specific proteins and their influence can be predicted [23].

\section{B. Bio-inspired Nanonetworking}

Besides bio-inspired networking solutions, communication on the nano-scale is being investigated. Despite the similarity between communication and network functional requirements of traditional and nano-scale networks, nanonetworks bring a set of unique challenges. In general, nano-machines can be categorized into two types: one type mimics the existing electromechanical machines and the other type mimics nature-made nano-machines, e.g., molecular motors and receptors. In both types, the dimensions of nano-machines render conventional communication technologies such as electromagnetic wave, acoustic, inapplicable at these scales due to antenna size and channel limitations. In addition, the available memory and processing capabilities are extremely limited, which makes the use of complex communication algorithms and protocols impractical in the nano regime [4].

Recent advances in this field have been essentially supported by findings in the bio-inspired networking domain. For example, molecular communication channels that have been modeled to study the concepts of this communication paradigm was first proposed as a result of an inspiration from cellular signaling networks. The derived results have also been shown to be directly used to investigate artificial molecular communication channels between nano-machines. This very young and vastly unexplored trans-disciplinary research field is an important artifact of bio-inspired networking. Further research is necessary to come up with more complete and realistic models describing the overall communication process, to analytically evaluate the performance of such communication channels, and to devise practical communication techniques following the main design principles of bio-inspired networking as outlined above.

\section{CONCLUSION}

In this article, the common fundamental networking challenges and the current status of research efforts to address them from the perspective of bio-inspired networking is captured. Through the existing research results, it has been shown that the inspiration from biology is, indeed, a powerful source of innovative network design. Despite the considerable amount of ongoing research, projects, journal special issues, conferences in this field, the bio-inspired networking research community is quite young, and there still remain significantly challenging tasks for the research community to address for the realization of many existing and most of the emerging networking architectures. With this regard, we outlined some open research questions that need to be targeted in the next years to foster both innovative and methodologically sound research. We believe that some of the most challenging questions in networking can only be solved in interdisciplinary teams tied to basic knowledge in engineering but also investigating unorthodox methods.

\section{REFERENCES}

[1] J. Timmis, M. Neal, and J. Hunt, "An Artificial Immune System for Data Analysis,” Biosystems, vol. 55, pp. 143-150, 2000.

[2] S. Camazine, J.-L. Deneubourg, N. R. Franks, J. Sneyd, G. Theraula, and E. Bonabeau, Self-Organization in Biological Systems. Princeton University Press, 2003.

[3] E. Bonabeau, M. Dorigo, and G. Theraulaz, Swarm Intelligence: From Natural to Artificial Systems. Oxford University Press, 1999.

[4] I. F. Akyildiz, F. Brunetti, and C. Blázquez, "Nanonetworks: A New Communication Paradigm," Elsevier Computer Networks, vol. 52, pp. 2260-2279, 2008 
[5] I. F. Akyildiz, O. B. Akan, C. Chen, J. Fang, and W. Su, "The state of the art in interplanetary Internet," IEEE Communications Magazine, vol. 42, no. 7, pp. 108-118, July 2004.

[6] F. Dressler, Self-Organization in Sensor and Actor Networks. John Wiley \& Sons, December 2007.

[7] M. Eigen and P. Schuster, The Hypercycle: A Principle of Natural Self Organization. Springer, 1979.

[8] F. Dressler and O. B. Akan, "A Survey on Bio-inspired Networking," Elsevier Computer Networks, vol. 54, no. 6, pp. 881-900, April 2010.

[9] M. Dorigo, V. Maniezzo, and A. Colorni, "The Ant System: Optimization by a colony of cooperating agents," IEEE Transactions on Systems, Man, and Cybernetics, vol. 26, no. 1, pp. 1-13, 1996.

[10] G. Di Caro and M. Dorigo, "AntNet: Distributed Stigmergetic Control for Communication Networks," Journal of Artificial Intelligence Research, vol. 9, pp. 317-365, December 1998.

[11] T. H. Labella and F. Dressler, "A Bio-Inspired Architecture for Division of Labour in SANETs," in 1st IEEE/ACM International Conference on Bio-Inspired Models of Network, Information and Computing Systems (BIONETICS 2006). Cavalese, Italy: IEEE, December 2006.

[12] L. N. de Castro and J. Timmis, Artificial Immune Systems: A New Computational Intelligence Approach. Springer, 2002.

[13] S. A. Hofmeyr and S. Forrest, "Architecture for an Artificial Immune System," Evolutionary Computation, vol. 8, no. 4, pp. 443-473, 2000.

[14] S. Stepney, R. E. Smith, J. Timmis, A. M. Tyrrell, M. J. Neal, and A. N. W. Hone, "Conceptual Frameworks for Artificial Immune Systems," International Journal of Unconventional Computing, vol. 1, no. 3, pp. 315-338, July 2005.

[15] B. Atakan and O. B. Akan, "Immune System Based Distributed Node and Rate Selection in Wireless Sensor Networks," in 1st IEEE/ACM International Conference on Bio-Inspired Models of Network, Information and Computing Systems (BIONETICS 2006). Cavalese, Italy: IEEE, December 2006

[16] G. Weng, U. S. Bhalla, and R. Iyengar, "Complexity in Biological Signaling Systems," Science, vol. 284, no. 5411, pp. 92-96, April 1999.

[17] T. Pawson, "Protein modules and signalling networks," Nature, vol. 373, no. 6515 , pp. 573-80, February 1995.

[18] R. Milo, S. Shen-Orr, S. Itzkovitz, N. Kashtan, D. Chklovskii, and U. Alon, "Network Motifs: Simple Building Blocks of Complex Networks," Nature, vol. 298, pp. 824-827, April 2002.

[19] F. Dressler, I. Dietrich, R. German, and B. Krüger, "A Rule-based System for Programming Self-Organized Sensor and Actor Networks," Elsevier Computer Networks, vol. 53, no. 10, pp. 1737-1750, July 2009.

[20] F. Dressler, "Bio-inspired Feedback Loops for Self-Organized Event Detection in SANETs," in 3rd IEEE/IFIP International Workshop on Self-Organizing Systems (IWSOS 2008), K. A. Hummel and J. Sterbenz, Eds., vol. LNCS 5343. Vienna, Austria: Springer, December 2008, pp. 256-261.

[21] C. Tschudin, "Fraglets - a Metabolistic Execution Model for Communication Protocols," in 2nd Symposium on Autonomous Intelligent Networks and Systems (AINS), Menlo Park, CA, June/July 2003.

[22] B. Webb, "What does robotics offer animal behaviour?" Animal Behavior, vol. 60 , no. 5 , pp. 545-558, 2000.

[23] N. D. Owens, J. Timmis, A. Greensted, and A. Tyrrell, "Elucidation of T cell signalling models," Journal of Theoretical Biology, vol. 262, no. 3, pp. 452-470, February 2010.

Falko Dressler (S'02, M'04, SM'08) is an assistant professor leading the Autonomic Networking Group at the Department of Computer Science, University of Erlangen. He teaches on self-organizing sensor and actor networks, network security, and communication systems. Dr. Dressler received his M.Sc. and Ph.D. degree from the Dept. of Computer Science, University of Erlangen in 1998 and 2003, respectively. Dr. Dressler is an Editor for journals such as Elsevier Ad Hoc Networks and ACM/Springer Wireless Networks (WINET). He was guest editor of special issues on self-organization, autonomic networking, and bio-inspired computing and communication for IEEE Journal on Selected Areas in Communications (JSAC), Elsevier Ad Hoc Networks, and others. Dr. Dressler was general chair of IEEE/ACM BIONETICS 2007 and IEEE/IFIP WONS 2011. Besides chairing a number of workshops associated to high-level conferences, he regularly acts in the TPC of leading networking conferences such as IEEE INFOCOM, IEEE ICC, IEEE Globecom, IEEE MASS, IFIP Networking and others. Dr. Dressler published two books including Self-Organization in Sensor and Actor Networks, published by Wiley in 2007. Dr. Dressler is a Senior Member of the IEEE (COMSOC, CS, VTS) as well as a Senior Member of ACM (SIGMOBILE), and member of GI (KuVS). He is actively participating in several working groups of the IETF. His research activities are focused on selforganizing networks addressing issues in wireless ad hoc and sensor networks, inter-vehicular communication systems, bio-inspired networking, and adaptive network security techniques.

Ozgur B. Akan (M'00-SM'07) received the B.S. and M.S. degrees in electrical and electronics engineering from Bilkent University and Middle East Technical University (METU), Ankara, Turkey, in 1999 and 2001, respectively. He received the Ph.D. degree in electrical and computer engineering from the Broadband and Wireless Networking Laboratory, School of Electrical and Computer Engineering, Georgia Institute of Technology, Atlanta, in 2004. He is currently Associate Professor with the Department of Electrical and Electronics Engineering, METU and the Director of Next-generation Wireless Communications Laboratory (NWCL). His current research interests are in wireless communications, bio-inspired communications, nano-scale and molecular communications, network information theory. Dr. Akan is an Associate Editor for IEEE Transactions on Vehicular Technology, International Journal of Communication Systems (Wiley), Nano Communication Networks Journal (Elsevier). He served as an Area Editor for AD HOC Networks Journal (Elsevier) (2004-2008), Editor for ACM/Springer Wireless Networks (WINET) Journal (2004-2010), as a Guest Editor for several special issues, as the TPC Co-Chair for The 13th ACM International Conference on Modeling, Analysis and Simulation of Wireless and Mobile Systems (ACM MSWiM 2010), the General Co-Chair for The Third International Conference on Bio-Inspired Models of Network, Information, and Computing Systems (ICST/IEEE BIONETICS 2008), the European Vice Chair for The Second International Conference on Nano-Networks (ICST/ACM Nano-Net 2007), an International Vice Chair for IEEE INFOCOM 2006, and in organizing and program committees of many other international conferences. He is the Vice President for IEEE Communications Society - Turkey Section. He is an IEEE Senior Member (Communications Society), and a member of ACM. Dr. Akan received the IBM Faculty Award twice in 2010 and 2008, Turkish Academy of Sciences Distinguished Young Scientist Award 2008 (TUBA-GEBIP). 